\title{
Decentralized Topology Reconfiguration in Multiphase Distribution Networks
}

\author{
Jingyuan Liu, Student Member, IEEE, Pirathayini Srikantha, Member, IEEE
}

\begin{abstract}
The cyber-physical nature of the modern power grid allows active power entities to exchange information signals with one another to make intelligent local actuation decisions. Exacting effective coordination amongst these cyber-enabled entities by way of strategic signal exchanges is essential for accommodating highly fluctuating power components (e.g. renewables, electric vehicles, etc.) that are becoming prevalent in today's electric grid. As such, in this paper, we present a novel decentralized topology reconfiguration algorithm for the distribution network (DN) that allows the system to adapt in real-time to unexpected perturbations and/or congestions to restore balance in loads across the feeder and improve the DN voltage profile. For this, individual agents residing in DN buses iteratively exchange signals with neighbouring nodes to infer the current state (e.g. power balance and voltage) of the system and utilize this information to make local line switching decisions. Strong convergence properties and optimality conditions of the proposed algorithm are established via theoretical studies evoking potential games and discrete concavity. Comparative simulation studies conducted on realistic DNs showcase the practical properties of the proposed algorithm.
\end{abstract}

\section{INTRODUCTION}

With the rapid proliferation of diverse power entities (e.g. loads, storage, distributed generation (DG), etc.) that induce highly variable power demand and supply patterns in the grid, maintaining secure system operation remains an open challenge for modern grid operators [1]. Existing grid infrastructure and power management processes are not engineered to accommodate high variability and uncertainty associated with these power entities. Upgrading the grid infrastructure will result in exorbitant costs. Not addressing these ongoing changes will lead to increased system vulnerabilities and stresses that will accumulate in costly failures and outages.

In this paper, we focus specifically on the distribution network (DN) where many of these diverse power components directly connect to. As these networks deliver power from the main grid to consumers, these are implemented in radial/tree configuration. Due to the high resistance to reactance ratio of DN power lines, significant loading of DN lines will result in voltage drops that can lead to cascading system outages [2]. Tie switches exist in DNs that can be utilized to reconfigure the physical DN network topology to restore balance in load/voltage distribution across the system. However, as the optimal DN topology reconfiguration problem is a combinatorial mixed-integer program consisting of nonlinear and non-convex constraints, computing an exact solution is generally NP-hard. Hence, this mechanism is not applied

J. Liu and P. Srikantha are with the Department of Electrical and Computer Engineering, Western University, London, ON, Canada; E-mails: jliu2325@uwo.ca and psrikan@uwo.ca. in real-time to relieve unexpected system congestions/stress caused by dynamically changing end nodes residing in the DN. In order to overcome these challenges, we leverage on the cyber-physical nature of the modern grid to propose a decentralized and adaptive DN topology reconfiguration algorithm [3]. The advanced metering infrastructure (AMI) and smart monitoring/control mechanisms widely integrated into today's DNs empower individual nodes such as buses and switches with the ability to communicate with one another and perform local computations [1]. We leverage on potential game theory to strategically design the content of signals exchanged amongst active nodes which provide unprecedented insights into the operational state of the DN. These signals are utilized by the cyber-enabled nodes to compute local actuation decisions (e.g. activation/deactivation of DN lines). This iterative signal exchange and actuation process is associated with strong convergence properties. Optimality conditions are derived using theoretical constructs from discrete concavity.

Existing work in the area of DN topology reconfiguration can be generally divided into three categories. Proposals belonging to the first category apply meta-heuristic techniques such as genetic algorithms [4], [5], ant colony optimization [6] and hybrid particle swarm optimization [7]. These metaheuristic approaches can be applied directly to the non-convex topology reconfiguration problem. However, these approaches also entail significant amount of computation time and highly granular parameter tuning with no guarantees on convergence speed and optimality. The second category of existing work apply convex relaxations for tractable computation. Methods of relaxation employed include MISOCP (Mixed Integer SecondOrder Cone Programming) [8], [9], [11], MILP (Mixed Integer Linear Programming) [12], QCP (Quadratically Constrained Programming) [9], MISDP (Mixed Integer Semi-Definite Programming) [13] and linearization of current flow [14]. These algorithms are implemented in a centralized manner by the electric power utility (EPU) that manages the DN. The EPU will need to process large volumes of data-sets generated in the DN over short intervals and this is not practical for real-time coordination. Moreover, these relaxations tend to omit important physical attributes of the DN (e.g. treating the system as a balanced single-phase system, ignoring reactive power, bus voltage, etc.). The third class of existing literature utilizes graph-theoretic methods to exploit structural properties of the DN to solve the topology reconfiguration problem. Hierarchical DN decomposition [15], branch exchange algorithm [16], best-first tree searching method [17] and open shortest path first (OSPF) [18] are examples of these proposals which offload part of the computational and communication 
tasks to several individual nodes that can act in parallel. These entail highly specific parameter tuning for supporting in-built heuristics which does not allow for generalization. Participating nodes also need to be continuously updated with conditions across the system for local decision-making and this is not scalable for real-time coordination.

As such, in this paper, we capitalize on the radial structure of the DN and the intrinsic relationship between loads and bus voltages to propose a novel distributed DN topology reconfiguration algorithm. Our contributions are five-fold as the proposed algorithm is: 1) Decentralized: Computational efforts are offloaded to agents representing DN buses/switches that compute their own decisions via lightweight signals exchanged with agents in adjacent buses; 2) Real-time: The algorithm is associated with very fast convergence speed and therefore is able to adapt to significant system fluctuations and congestions within seconds; 3) Scalable: The algorithm scales well to the size of the DN and the number of tie switches present; 4) Efficient: Conditions for optimality have been established via theoretical constructs from potential games and discrete concavity; and 5) Practical: Important physical attributes that include the unbalanced multiphase nature of a real DN and system states such as reactive power and bus voltage magnitudes are considered. We highlight these contributions via comprehensive theoretical studies and simulations conducted on practical DNs along with comparisons with state-of-the-art.

The remainder of this paper is organized as follows. In Sec. II, the system model, notations utilized in the paper and DN topology reconfiguration problem are introduced. The proposed decentralized algorithm is presented in Sec. III along with theoretical analysis. Practical simulations and comparisons with existing work are presented in Sec. IV. The paper is concluded in Sec. V.

\section{SySTEM MODEL}

In this section, we present a detailed overview of the system settings, assumptions, and notations utilized to represent a cyber-enabled low-voltage multiphase DN. We, then, present the DN topology reconfiguration problem and highlight the challenges associated with solving it.

\section{A. System Settings and Assumptions}

For the system settings, a DN is defined as a radial network (i.e. acyclic) mainly composed of lines (i.e. edges) that transport power from the substation/feeder (connecting to the bulk power grid) to buses (i.e. vertices/nodes) where loads and micro-generation systems attach to. The DN considered in this paper is composed of buses that are each equipped with monitoring, computational and communication capabilities. These bus modules can actuate local switches located in directly connected lines to activate or deactivate these as necessary. This cyber-physical nature of the DN is in line with the smart grid vision of the electricity sector [3]. Next, we consider the DN to be a low-voltage multiphase system which is a more general system model than existing state-of-theart that treats the DN as a single-phase balanced system [2]. Finally, due to recent advances in phase balancing algorithms
[19], [20] and analyses from references such as [21], transience resulting from the activation/deactivation of lines in the DN is not considered to introduce adverse effects to the system. From the aforementioned system settings, advances in smart grid technologies and current state-of-the-art, we make the following assumptions about the DN:

1) Voltage magnitude constraints on $\mathrm{DN}$ buses are assumed to be $1.0 \pm 0.05$ p.u.;

2) Local voltage control devices (e.g. voltage regulators and capacitor banks) are inactive during the reconfiguration process; and

3) The overall power drawn by each bus in the DN is assumed to be constant over five-second intervals;

For Assumption 1, we adopt the voltage constraints typically maintained by DN system operators where the bus voltage magnitudes are allowed to deviate around 0.05 p.u. around the nominal value of 1 p.u. $(1.0 \pm 0.05$ p.u. $)$. This is more conservative than the actual tolerance margin of $1.0 \pm 0.1$ p.u. prior to equipment failures in DNs [2]. These conservative bounds can accommodate minor deviations in voltage computed using the three-phase model utilized in this paper from the actual values as discussed in Sec. II-B. Thus, both reverse power flows resulting from the proliferation of renewable energy sources and battery energy management systems (BEMS) [1], [22] and high power consumption from large residential appliances such as electric vehicles can be readily accommodated by our model. With Assumption 2, we are rendering the voltage control schemes of devices such as voltage regulates and capacitor banks inactive as we are focussing on topology reconfiguration of the DN in this paper. In future work, we will investigate how these devices can be combined with topology reconfiguration for increased resilience and efficiency in the DN. Assumption 3 allows for greater accuracy in modelling fluctuations in load profiles in the DNs which are composed of increasingly variable components (e.g. distributed generation, etc.). Existing proposals on topology reconfiguration (e.g. [6], [24]) are designed over longer time scales that range from 10 minutes to yearly intervals. These long forecast horizons are typically associated with greater margins of error especially with distributed energy sources as demonstrated in reference [23]. Thus, the short coordination interval of five seconds considered in this paper eliminates these forecast errors.

\section{B. Multiphase Power Flow Relations and Notations}

Next, we introduce the multiphase power flow relations adopted from reference [10] and notations utilized in this paper to model interdependencies of physical states such as voltage, power flow and current flow in the low-voltage DN. Variables are denoted by capital fonts, and system parameters are denoted by small fonts. All buses in the DN form the set $\mathcal{B}$. Currently active DN lines form the set $\mathcal{E}$ where $(r, l) \in \mathcal{E}$ denotes an active line connecting bus $r$ to bus $l$. Bus $l$ is closer to the feeder than bus $r$ in terms of the length of the path between the bus and the feeder. The maximum number of phases any DN bus/line can have is three and these are labelled as $a, b$ and $c$. The phases associated with bus $r \in \mathcal{B}$ and line $(r, l) \in \mathcal{E}$ are represented by the sets $\Phi_{r}$ and $\Phi_{r l}$ respectively. 
The relation between these phase sets is $\Phi_{l} \supseteq \Phi_{r l}=\Phi_{r}$ given that $(r, l) \in \mathcal{E}$. The complex impedance $z_{r l}$ of line $(r, l)$ is a full-rank $3 \times 3$ matrix. Impedances of all lines present in the DN form the set $\mathcal{Z}$ where $z_{r l} \in \mathcal{Z}$. The topology $\mathcal{T}$ of the DN is completely defined by $\{\mathcal{B}, \mathcal{E}, \mathcal{Z}\}$.

The voltage $V_{r}$ of bus $r$ consists of components $\left[V_{r}^{\phi} \in\right.$ $\mathbb{C}]_{\phi \in \Phi_{r}}$ where $V_{r}^{\phi}$ represents the voltage of the individual phase $\phi \in \Phi_{r}$. Superscripts are used to denote the projection of a system state to specific phases and non-existing phase entries are filled with 0 . For instance, consider a bus $r$ for which $\Phi_{r}=a b$. Then, the voltage $V_{r}$ is $\left(V_{r}^{a}, V_{r}^{b}\right)^{T}$. If this is to be projected to $\phi_{l}=a b c$, then $V_{r}^{\Phi_{l}}=\left(V_{r}^{a}, V_{r}^{b}, 0\right)^{T}$. The current flow $I_{r l}$ on line $(r, l) \in \mathcal{E}$ is a vector consisting of $\left|\Phi_{r l}\right|$ elements. The Ohm's law relates voltage drop and current flow on line $(r, l)$ :

$$
V_{l}^{\Phi_{r l}}=V_{r}-z_{r l} I_{r l}
$$

The overall current injected by bus $r$ is $I_{r}=\left[I_{r}^{\phi} \in \mathbb{C}\right]_{\phi \in \Phi_{r}}$. The complex power injected by bus $r$ is defined as $S_{r}=\left[S_{r}^{\phi} \in \mathbb{C}\right]_{\phi \in \Phi_{r}}$ which can be computed using voltage and current variables:

$$
S_{r}=\operatorname{diag}\left(V_{r} I_{r}^{H}\right)
$$

where diag is the notation used to represent diagonal elements of a matrix in vector form and the operator $H$ is the Hermitian transpose. It is clear that Eq. 1 is linear in terms of voltage and current variables. However, Eq. 2 represents power balance and is not linear in terms of the state variables and thus is a non-convex physical constraint. This non-convex quadratic equality constraint and the integer set that represents feasible network topology configurations render the associated network topology reconfiguration problem a non-convex combinatorial problem that is NP-Hard as indicated by reference [11].

Thus, in order to avoid this issue, artificial variables $\hat{V}_{r}=$ $V_{r} V_{r}^{H}, \hat{I}_{r l}=I_{r l} I_{r l}^{H}$ and $S_{r l}=V_{r}^{\Phi_{r l}} I_{r l}^{H}$ are introduced and these are matrices of sizes $\left|\phi_{r}\right| \times\left|\phi_{r}\right|$ and $\left|\phi_{r l}\right| \times\left|\phi_{r l}\right|$ respectively. The diagonal elements of $\hat{V}_{r}$ and $\hat{I}_{r l}$ represent the square voltage magnitude of bus $r$ and square magnitude of current flowing on line $(r, l)$. Similarly, the diagonal elements of $S_{r l}$ represent the power flow from bus $r$ to $l$ across the phases $\Phi_{r l}$. Power balance can be defined as follows using the artificial variables:

$$
\sum_{q:(q, r) \in \mathcal{E}} \operatorname{diag}\left(S_{q r}-z_{q r} \hat{I}_{q r}\right)+S_{r}=\sum_{l:(r, l) \in \mathcal{E}} \operatorname{diag}\left(S_{r l}\right)^{\Phi_{r}}
$$

The first term encapsulates the total power flowing from direct descendants $q$ of bus $r$. The second term incorporates power loss due to the impedance of the lines $(q, r) \in \mathcal{E}$. The third term denotes power injection into bus $r$. The final term represents power flowing out of bus $r$. It is clear that Eq. 3 is a linear relation in terms of the artificial variables. In order to express the voltage drop relation in terms of these artificial variables, the Hermitian transpose of Eq. 1 is multiplied on both sides to obtain:

$$
\hat{V}_{l}^{\Phi_{r l}}=\hat{V}_{r}-\left(S_{r l} z_{r l}{ }^{H}+z_{r l} S_{r l}^{H}\right)+z_{r l} \hat{I}_{r l} z_{r l}{ }^{H}
$$

Although Eq. 4 is a linear relation, $S_{r l}$ is still a nonlinear function of $I_{r l}$ and $\hat{V}_{r}$. Reference [10] overcomes this issue by first assuming that $z_{r l} \hat{I}_{r l} \ll S_{r l}$ which eliminates the $z_{q r} \hat{I}_{q r}$ term in Eq. 3 and $z_{r l} \hat{I}_{r l} z_{r l}{ }^{H}$ term in Eq. 4. Upon examining Eq. 4 it is clear that ignoring power loss will result in the difference in the magnitude of voltages across buses being higher than incorporating it. Reference [10] has demonstrated that the difference in actual voltage magnitudes and the values computed via the model utilized in this paper is 0.0016 p.u. in DNs (i.e. IEEE 33-bus, IEEE 34bus, IEEE 37-bus, IEEE 123-bus and Rossi 2056-bus systems) located in Southern California which is a state associated with significant penetration of renewable generation sources (e.g. photovoltaics). Thus, this minor deviation of 0.0016 p.u. is much lower than the tolerance margin of \pm 0.05 p.u. available prior to the onset of equipment failures in the DN. Next, it is assumed that bus voltages across the phases are nearly balanced. This implies:

$$
V_{r}^{a} \approx V_{r}^{b} e^{\mathbf{j} 2 \pi / 3} ; V_{r}^{b} \approx V_{r}^{c} e^{\mathbf{j} 2 \pi / 3} ; V_{r}^{c} \approx V_{r}^{a} e^{\mathbf{j} 2 \pi / 3}
$$

when $\Phi_{r}=a b c$ where $\mathbf{j}$ is the imaginary number $\sqrt{-1}$. The above set of relations imply that the phase angle difference is approximately $120^{\circ}=2 \pi / 3$ radians. Suppose that $\Lambda_{r l}$ is a vector whose elements are the diagonal components of $S_{r l}$ that represent the power flow on each phase of line $(r, l)$, then it is possible to define all elements of $S_{r l}$ in terms of $\Lambda_{r l}$ :

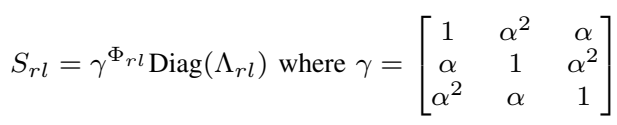

where the Diag operator creates a matrix whose diagonal components are $\Lambda_{r l}$ and off-diagonal elements are 0 . These relations that represent the underlying physical infrastructure dependencies are now linear. These convex relations are able to preserve important system attributes (e.g. multiphase nature of DN) and variables (e.g. bus voltage, real/reactive power, etc.). Comprehensive accuracy analyses conducted by reference [10] demonstrate that these relaxations result in negligible error (i.e. within 0.0016 per unit (p.u.) of true values) for practical DNs. Therefore, these approximations allow for conservative decision-making as mentioned earlier.

Next, specific graph notations utilized in the proposed algorithm are introduced next. As we consider an iterative algorithm for topology reconfiguration (presented in Sec. III), these notations are based on the currently active DN topology. We omit the labelling of the iteration number of the algorithm for simplification of the notations. Current path formed between bus $r$ and its ancestor bus $b$ is represented by the set $\mathcal{E}_{r b}$ which consists of edges in $\mathcal{E}$ that form this path. The lowest common ancestor of buses $a$ and $b$ for the present topology referenced from the feeder is denoted as $A_{a, b}$. The set $\mathcal{B}_{r}$ denotes the subtree rooted at bus $r$ and is composed of all buses this subtree currently contains. $\mathcal{B}_{r}$ is composed of buses in the set $\mathcal{B}_{r} \backslash r$. $\mathcal{L}_{r}$ consists of buses that are leaves of the subtree $\mathcal{B}_{r} . P_{r}$ is the current parent of bus $r$. All buses capable of switching (i.e. reconfiguration) form the set $\mathcal{R}$. A bus $r \in \mathcal{R}$ is associated with a set of potential parents $\mathcal{P}_{r}$. Bus $r$ can form an edge with any node in $\mathcal{P}_{r}$ and the number of phases supported by the potential parent nodes must be a superset of bus $r$. If bus $r$ 's parent is currently bus $l \in \mathcal{P}_{r}$, then 
$(r, l) \in \mathcal{E}$ and $z_{r l} \in \mathcal{Z}$. If bus $r$ decides to switch to another node $u \in \mathcal{P}_{r}$, then a new edge $(r, u)$ and impedance $z_{r u}$ will be added to $\mathcal{E}$ and $\mathcal{Z}$ respectively while the original edge $(r, l)$ and impedance $z_{r l}$ will be removed from the corresponding sets.

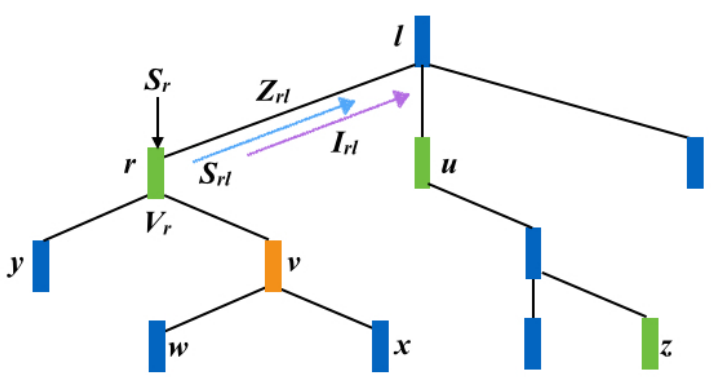

Fig. 1: Illustration of notations in a radial network.

These notations are illustrated in a simple radial network presented in Fig. 1 where bus $l$ is the feeder node. This bus is also $P_{r}$ as it is the parent of node $r$. Although line attributes $z_{r l}, S_{r l}, I_{r l}$ are illustrated for a single edge, these exist for all edges $(r, l) \in \mathcal{E}$. Similarly, bus attributes $S_{r}, V_{r}$ apply to all buses $b \in \mathcal{B}$. For ease of pictorial depiction, the multiphase nature of the DN lines is implied by single edges. Buses composing the subtree rooted at bus $r$ are represented by $\mathcal{B}_{r}=\{r, y, v, w, x\}$, the leaves of this subtree are $\mathcal{L}_{r}=\{y, w, x\}$ and $\mathcal{B}_{r}$ is $\{y, v, w, x\}$. The path from bus $v$ to $l$ is $\mathcal{E}_{v l}=\{(v, r),(r, l)\}$. The lowest common ancestor $A_{v u}$ of buses $v$ and $u$ is bus $l$. Node $v$ is the only reconfigurable bus in this example and therefore $\mathcal{R}=\{v\}$. The potential parents of bus $v$ are contained in $\mathcal{P}_{v}=\{r, u, z\}$.

\section{Topology Reconfiguration Problem Formulation}

The DN topology reconfiguration problem $\mathcal{P}_{T R}$ presented next aims to improve the voltage profile of a low-voltage multiphase DN in the presence of highly fluctuating power components while accounting for physical grid constraints.

$$
\begin{array}{lr}
\mathcal{P}_{T R}: & \\
\min _{\mathcal{T}(\mathcal{R}, \mathcal{P})} F(\mathcal{T}):=\sum_{r \in \mathcal{B}} \sum_{\phi \in \Phi_{r}}\left(\hat{V}_{f}^{\phi}-\hat{V}_{r}^{\phi}\right) \text { s.t. } \\
\quad \sum_{m:(m, r) \in \mathcal{E}} \Lambda_{m r}^{\Phi_{r}}+S_{r}=\Lambda_{r l} & r \in \mathcal{B} \backslash f \\
S_{r l}=\gamma^{\Phi_{r l}} \operatorname{Diag}\left(\Lambda_{r l}\right)=P_{r l}+\mathbf{j} Q_{r l} & (r, l) \in \mathcal{E} \\
\left(P_{r l}^{\phi}\right)^{2}+\left(Q_{r l}^{\phi}\right)^{2} \leq \bar{s}_{r l}^{2} & \phi \in \Phi_{r l},(r, l) \in \mathcal{E} \\
\hat{V}_{r}-\hat{V}_{l}^{\Phi_{r l}}=z_{r l} S_{r l}^{H}+S_{r l} z_{r l} H & (r, l) \in \mathcal{E} \\
S_{r}=P_{r}+\mathbf{j} Q_{r} & r \in \mathcal{B} \\
\underline{p}_{r} \leq P_{r}^{\phi} \leq \bar{p}_{r}, \underline{q}_{r} \leq Q_{r}^{\phi} \leq \bar{q}_{r} & \phi \in \Phi_{r}, r \in \mathcal{B} \\
\underline{v}_{r} \leq \hat{V}_{r}^{\phi} \leq \bar{v}_{r} & \phi \in \Phi_{r}, r \in \mathcal{B}
\end{array}
$$

The objective function $F(\mathcal{T})$ of $\mathcal{P}_{T R}$ consists of $\hat{V}_{r}^{\phi}$ terms which represent the square of the voltage magnitude of phase $\phi$ in bus $r$ (i.e. $\operatorname{diag}\left(\hat{V}_{r}\right)[\phi]$ ). Thus, the problem formulation minimizes the square magnitude voltage drop of every phase in all DN buses with respect to the feeder bus at the root of the radial distribution network. The feeder bus is a slack bus that connects to the high voltage transmission network. As such, it maintains a constant voltage magnitude $\left|V_{f}\right|=1$ p.u. across all phases and $\Phi_{f}=a b c$. This voltage profile optimizing criterion is inspired by existing literature in minimal voltage regulation(e.g. [5], [29]) and decentralized optimization for voltage control (e.g. [30]). The optimization variable is the DN topology $\mathcal{T}$ which is defined by $\mathcal{B}, \mathcal{E}$ and $\mathcal{Z}$. The topology construction is subject to discrete constraints imposed by $\mathcal{R}$ and $\mathcal{P}=\left\{P_{r}: \forall r \in \mathcal{R}\right\}$ along with convex steady-state power flow constraints listed in Eqs. 7 to 10 and system limits specified in Eqs. 11 to 13. Eqs. 7 and 8 impose power balance across all buses. $P_{r l}$ and $Q_{r l}$ in Eq. 8 represent real and imaginary components of $S_{r l}$ and these are matrices of size $\left|\Phi_{r l}\right| \times\left|\Phi_{r l}\right|$. Eq. 9 represents apparent power flow limit of each phase $\phi \in \Phi_{r l}$ where $\bar{s}_{r l}$ is the upper threshold parameter defined for line $(r, l)$. Eq. 10 relates voltage drop to the power flow in line $(r, l)$. Eq. 11 represents power injection by bus $r$ in terms of its real $P_{r}$ and reactive $Q_{r}$ components which are vectors of size $\left|\Phi_{r}\right| . S_{r}$ is determined by the composition of real/reactive loads and power injecting sources present in bus $r$. Eq. 12 imposes upper and lower limits on real and reactive power injection of each phase present in $\mathrm{DN}$ buses. The final constraint in Eq. 13 instills bounds on the square voltage magnitude corresponding to each phase in a bus.

$\mathcal{P}_{T R}$ represents a real-time optimization problem as consumer demands are assumed to remain constant over 5 second intervals. This renders $S_{r}$ a highly fluctuating parameter. Thus, $\mathcal{P}_{T R}$ must be solved within this short time interval in order to meet real-time requirements. Directly solving $\mathcal{P}_{T R}$ is challenging mainly due to the discrete nature of the topology formation problem which is further complicated by the highly coupled nature of Eqs. 7 to 10 . These challenges prevent the straightforward application of decentralized convex optimization techniques for solving $\mathcal{P}_{T R}$.

\section{DeCENTRALIZED DN TOPOLOGY Formation}

In order to construct a decentralized topology reconfiguration algorithm that allows for real-time computations, the challenges outlined in the previous section must be overcome. For this, we leverage on the cyber-physical nature of the DN that allow bus agents to measure local system states (e.g. voltage, current, power flow) and to exchange these states amongst neighboring agents in the network. This peer-topeer signal exchange allows us to infer the impact of a local switching decision on the network and utilize this information to iteratively compute local line switching for voltage profile balancing. This process is modelled in Fig. 2 and motivated by recent work in references [32] and [33] that focus on multiagent systems for distributed management in the power grid.

Each decision-making entity in the $\mathrm{DN}$ is a bus agent that is supplied local state measurements via monitoring devices such as micro phasor measurement units which provide information about the local environment (e.g. voltage, current, power flow, etc.) and signals communicated by peer nodes that reveal information about the external environment (i.e. general state of optimality and room for improvement in efficiency). The underlying physical network determines the bus agent's 


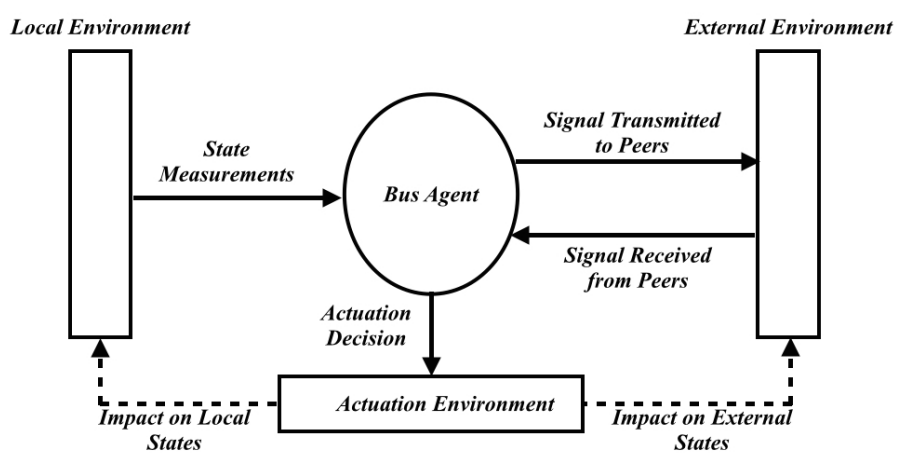

Fig. 2: Adaptive topology reconfiguration.

peers (i.e. that are graphically adjacent). The bus agent also summarizes its local conditions in signals and broadcasts these to peers. Thus, local actuation decisions made by each bus agent are dependent on these signals exchanged amongst peers along with local state measurements. Local actuation entails the activation and deactivation of DN lines that connect to the bus agent and this has an impact on both the external states (e.g. change in efficiency, etc.) and local states (i.e. power flow, bus voltage magnitudes, etc.). In the proposed topology reconfiguration algorithm, we strive to design the content of the signals exchanged amongst peers and local decision-making by individual bus agents. Strategic design of signals and actuation over the DN system will allow for the efficient progression of the proposed distributed iterative algorithm to desired states (i.e. Nash Equilibrium, optimality, etc.).

As such, in our proposal, every bus agent $r \in \mathcal{R}$ will select a random exponentially distributed time to perform local actuation (i.e. switch to a bus in $\mathcal{P}_{r}$ if necessary). It is highly unlikely for more than one bus agent to perform a local actuation at the same time due to the continuous and independent nature of the random process used to select the strategy revision time. Therefore, we can safely deduce the impact of actuation by a bus agent on the system by assuming that the remainder of the DN remains stationary (also supported by the five second constant demand intervals). This also allows buses in the DN to actuate without synchronization and knowledge of the entire system [31]. When its turn has arrived to make a strategy revision, bus agent $r$ evaluates its actuation strategies in $\mathcal{P}_{r}$ via local state measurements (e.g. bus voltage $\hat{V}_{r}$ and bus load $S_{r}$ ) and signals transmitted by peers (discussed later in this section). These provide insights regarding the local and external environment. Once every strategy in $\mathcal{P}_{r}$ is evaluated, $r$ proceeds to selecting the strategy that most reduces the overall cost incurred by the system.

If strategy revisions made by every bus agent reduces the global cost of the entire $\mathrm{DN}$, then this results in a potential game which is entirely defined by the players (i.e. bus agents $r \in \mathcal{B}$ ), discrete strategies (i.e. line activation/deactivation determined by $\mathcal{P}_{r}$ ) and cost (i.e. impact on the system). At equilibrium, no bus agent will deviate from their local strategies as this can result in greater costs (i.e. regret) and this state is referred to as the Nash Equilibrium. We also show that the Nash Equilibrium is also the globally optimal solution of $\mathcal{P}_{T R}$ when discrete concavity conditions are met. Prior to discussing the convergence and optimality properties of the proposed algorithm, we present the computation of the impact/cost on the system of a local line switching decision made by a bus agent using locally available information and peer-to-peer signal exchanges. We generalize this computation to four specific cases which covers all possible conditions that an actuating bus agent in the DN can be subject to.

Our previous works (e.g. [40]-[42]) like this paper have capitalized on the cyber-physical nature of the power grid as outlined in Fig. 2. However, main differences lie in the applications considered along with the underlying theoretical methods utilized to construct the signals exchanged and actuation decisions made by cyber-physical grid agents. Specifically, in our previous work, we focussed on optimal power flow via demand response and dispatch of distributed generation systems unlike this paper where we consider DN topology reconfiguration. While the optimal power flow problem consists of non-convexities, the topology reconfiguration problem introduces an additional layer of complexity due to the discrete variable space (i.e. line switching). Thus, the underlying theoretical constructs utilized for designing signal exchanges and decision-making in this paper are based on discrete concavity and potential games while our previous works capitalized on population game theoretic constructs that depend on convex formulations in the continuous domain. Moreover, unlike our previous works, the proposed DN topology reconfiguration algorithm is completely decentralized as no central entity is present to guide the bus agents in the decision-making process.

\section{A. Quantification of Line Switching Impact}

The cost function is designed to assimilate the impact of the actuation decision made by bus $r \in \mathcal{R}$ on the voltage profile of the system. Insights from $\mathcal{P}_{T R}$ are leveraged for the construction of this cost function. We first note that the overall voltage magnitude drop from the feeder node $f$ to any of its descendent $h$ across all the active phases associated with bus $h$ can be expressed as:

$$
\sum_{\phi \in \Phi_{h}}\left(\hat{V}_{f}^{\phi}-\hat{V}_{h}^{\phi}\right)=\sum_{(m, r) \in \mathcal{E}_{h, f}} \sum_{\phi \in \Phi_{h}}\left(\hat{V}_{r}^{\phi}-\hat{V}_{m}^{\phi}\right)
$$

which is a summation of voltage drops incurred across edges forming the path between $f$ and $h$ across all active phases associated with bus $h$. Summing the change in bus voltage magnitudes across the phases can result in obscuring differences present in specific phases. However, as we are enforcing stricter bus voltage magnitude limits on each phase (i.e. Eq. 13), these excursions will not result in significant deviations in bus voltage magnitudes across the phases. Relating voltage drop to power flow as specified in Eq. 10 yields $\sum_{\phi \in \Phi_{h}}\left(\hat{V}_{r}-\hat{V}_{m}\right)=\sum_{\phi \in \Phi_{h}}\left(z_{m r}{ }^{H} S_{m r}+S_{m r}^{H} z_{m r}\right)^{\phi}$. If the cumulative power consumption in subtree $\mathcal{B}_{m}$ is defined as $C_{m}=\gamma^{\Phi_{f}} \operatorname{Diag}\left(\sum_{r \in \mathcal{B}_{m}} S_{r}^{\Phi_{f}}\right)$, then $S_{m r}=C_{m}$ based on the approximation applied in Sec. II-B. Thus, the aforementioned voltage drop can be established as: 


$$
\sum_{\phi \in \Phi_{h}}\left(\hat{V}_{f}^{\phi}-\hat{V}_{h}^{\phi}\right)=-\sum_{(m, r) \in \mathcal{E}_{h, f}} \sum_{\phi \in \Phi_{h}}\left(z_{m r}{ }^{H} C_{m}+C_{m}^{H} z_{m r}\right)^{\phi}
$$

When bus $i$ makes a switch from node $j_{i} \in \mathcal{P}_{i}$ to $k_{i} \in \mathcal{P}_{i}$, voltage magnitudes will change for certain buses. From Eq. 15 , it is evident that changes in voltage drop are dependent on $C_{i}$ and the specific part of the DN network where the reconfiguration is taking place. Specifically, the manner in which the voltage magnitudes change can be separated into four cases as illustrated in Fig. 3. The lines in which power flow changes due to the switch made by bus agent $i$ from the original parent $j_{i}$ to the new parent $k_{i}$ are marked in red.

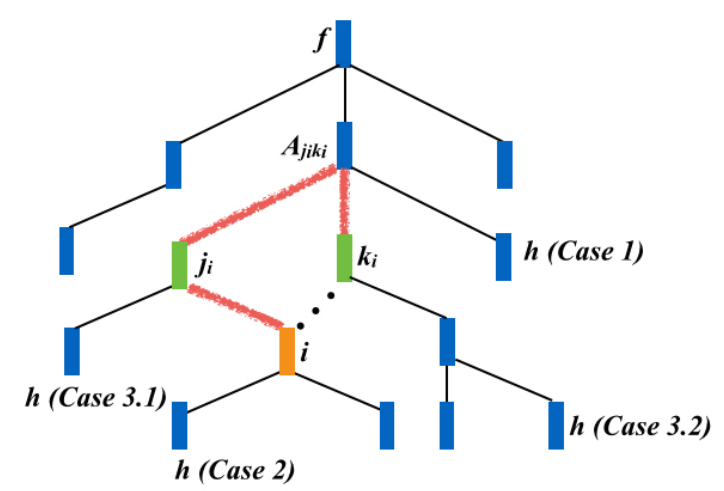

Fig. 3: Impact of a switch in the DN.

Since the power flowing in lines belonging to the path $\mathcal{E}_{A_{j_{i} k_{i} f}}$ will not change due to the switch, voltage magnitudes of buses $h \notin \mathcal{B}_{A_{j_{i} k_{i}}}$ will not be affected. This represents Case 1. The remaining three cases are relevant to all nodes $h \in \mathcal{B}_{A_{j_{i} k_{i}}}$ as the path $\mathcal{E}_{h f}$ from node $h$ to the feeder contains lines in which power flow has changed in proportion to $C_{i}$. Specifically, the ensuing voltage change (i.e. $\Delta$ ) can be expressed as:

$$
\sum_{\phi \in \Phi_{h}} \Delta\left(\hat{V}_{f}^{\phi}-\hat{V}_{h}^{\phi}\right)=\sum_{\phi \in \Phi_{h}} \Delta\left(\hat{V}_{A_{j_{i} k_{i}}}^{\phi}-\hat{V}_{h}^{\phi}\right) \quad \forall h \in \mathcal{B}_{A_{j_{i} k_{i}}}
$$

It is possible to further simplify the computation of the voltage change by considering the specific location of node $h$ within the subtree $\mathcal{B}_{A_{j_{i} k_{i}}}$. This leads to two general cases: Case 2) $h$ belongs to the subtree rooted at the switching node $i$ (i.e. $h \in B_{i}$ ); or Case 3) $h$ does not belong to the $\mathcal{B}_{i}$.

In Case 2, there will be no change in the cumulative power drawn by nodes residing within the subtree $\mathcal{B}_{i}$ as we fix power consumption over the optimization interval of 5 seconds. Thus, from Eq. 16 we can conclude that the change in voltage magnitude drop between the switching node $i$ and any of its descendants will be $\sum_{\phi \in \Phi_{h}} \Delta\left(\hat{V}_{i}^{\phi}-\hat{V}_{h}^{\phi}\right)=0$. The computation of Eq. 16 for $h \in \mathcal{B}_{i}$ can now be further decomposed into three components: 1) Change in the original voltage terms of the old and new parent of bus $i$ prior to the switch (i.e. $\left.\sum_{\phi \in \Phi_{h}}\left(\hat{V}_{j_{i}}^{\prime \phi}-\hat{V}_{k_{i}}^{\prime \phi}\right)\right)$, 2) Voltage difference due to the change in impedance from the old edge $\left(i, j_{i}\right)$ to the new edge $\left(i, k_{i}\right)$ (i.e. $-\sum_{\phi \in \Phi_{h}}\left[\left(z_{i k_{i}}{ }^{H}-z_{i j_{i}}{ }^{H}\right) C_{i}+\right.$ $\left.\left.C_{i}^{H}\left(z_{i k_{i}}-z_{i j_{i}}\right)\right]^{\phi}\right)$, and 3) Change in voltage from the common ancestor $A_{j_{i} k_{i}}$ to $k_{i}$ due to the difference in cumulative power flow of $C_{i}$ in these lines (i.e. $\sum_{\phi \in \Phi_{h}} \Delta\left(\hat{V}_{A_{j_{i} k_{i}}}\right.$ $\left.\left.\hat{V}_{k_{i}}^{\phi}\right)=-\sum_{(r, l) \in \mathcal{E}_{k_{i}, A_{j_{i} k_{i}}}} \sum_{\phi \in \Phi_{h}}\left(z_{r l}{ }^{H} C_{i}+C_{i}^{H} z_{r l}\right)^{\phi}\right)$. Combining these elements together results in for $h \in \mathcal{B}_{i}$ :

$$
\begin{aligned}
& \sum_{\phi \in \Phi_{h}} \Delta\left(\hat{V}_{f}^{\phi}-\hat{V}_{h}^{\phi}\right)=\sum_{\phi \in \Phi_{h}}\left\{\left(\hat{V}_{j_{i}}^{\prime \phi}-\hat{V}_{k_{i}}^{\prime \phi}\right)-\left[\left(z_{i k_{i}}{ }^{H}-z_{i j_{i}}{ }^{H}\right) C_{i}\right.\right. \\
& \left.\left.+C_{i}^{H}\left(z_{i k_{i}}-z_{i j_{i}}\right)\right]^{\phi}-\sum_{(r, l) \in \mathcal{E}_{k_{i} A_{j_{i} k_{i}}}}\left(z_{r l}{ }^{H} C_{i}+C_{i}^{H} z_{r l}\right)^{\phi}\right\}
\end{aligned}
$$

For the next case, where $h \notin \mathcal{B}_{i}, h$ can be a member of the subtree rooted at the immediate descendant node of $\mathcal{B}_{A_{j_{i} k_{i}}}$ that contains the old parent node $j_{i}$ (Case 3.1). It can also belong to the subtree rooted at another descendent node of $\mathcal{B}_{A_{j_{i} k_{i}}}$ that contains the new parent node $k_{i}$ (Case 3.2). In the first sub-case, there is a net decrease in power flow in the edges belonging to the path $\mathcal{E}_{A_{j_{i} h} A_{j_{i} k_{i}}}$ by $C_{i}$ which leads to:

$$
\sum_{\phi \in \Phi_{h}} \Delta\left(\hat{V}_{f}^{\phi}-\hat{V}_{h}^{\phi}\right)=\sum_{(r, l) \in \mathcal{E}_{A_{j_{i} h} A_{j_{i} k_{i}}}} \sum_{\phi \in \Phi_{h}}\left(z_{r l}{ }^{H} C_{i}+C_{i}^{H} z_{r l}\right)^{\phi}
$$

In the second sub-case, there is a net increase in power flow of $C_{i}$ in the edges of the path $\mathcal{E}_{A_{k_{i} h} A_{j_{i} k_{i}}}$ which leads to:

$$
\sum_{\phi \in \Phi_{h}} \Delta\left(\hat{V}_{f}^{\phi}-\hat{V}_{h}^{\phi}\right)=-\sum_{(r, l) \in \mathcal{E}_{A_{k_{i} h} A_{j_{i} k_{i}}}} \sum_{\phi \in \Phi_{h}}\left(z_{r l}{ }^{H} C_{i}+C_{i}^{H} z_{r l}\right)^{\phi}
$$

From Eq. 17-19, we know exactly how the voltage magnitude of every $h \in \overline{\mathcal{B}_{A_{j_{i} k_{i}}}}$ will be affected by the switching of $i$. This allows us to formulate the cost function in a decentralized manner as only one bus $i \in \mathcal{R}$ performs a switch from node $j_{i}$ to $k_{i}$ at any time instant. The topology of the DN is denoted as $\mathcal{T}^{j_{i}}$ when the edge $(i, j)$ exists and $\mathcal{T}^{k_{i}}$ when the edge $(i, k)$ exists (i.e. prior to and after a switch). The change in the overall cost $f_{j_{i} k_{i}}$ of the system due to $i$ switching from $j_{i}$ to $k_{i}$ is:

$$
\begin{aligned}
& f_{j_{i} k_{i}}=F\left(\mathcal{T}^{j_{i}}\right)-F\left(\mathcal{T}^{k_{i}}\right)= \\
& \sum_{\phi \in \Phi_{f}}\left\{\left(\hat{V}_{k_{i}}^{\prime}-\hat{V}_{j_{i}}^{\prime}+\left(\left(z_{i k_{i}}{ }^{H}-z_{i j_{i}}{ }^{H}\right) C_{i}+C_{i}^{H}\left(z_{i k_{i}}-z_{i j_{i}}\right)\right)\right)^{\phi}\left|\mathcal{B}_{i}^{\phi}\right|\right. \\
& +\sum_{(r, l) \in \mathcal{E}_{j_{i} A_{j_{i} k_{i}}}}\left(\left(z_{r l}{ }^{H} C_{i}+C_{i}^{H} z_{r l}\right)^{\phi}\left(\left|\mathcal{B}_{r}{ }^{\phi}\right|-\left|\mathcal{B}_{i}^{\phi}\right|\right)\right) \\
& \left.-\sum_{(r, l) \in \mathcal{E}_{k_{i} A_{j_{i} k_{i}}}}\left(\left(z_{r l}{ }^{H} C_{i}+C_{i}^{H} z_{r l}\right)^{\phi}\left(\left|\mathcal{B}_{r}{ }^{\phi}\right|+\left|\mathcal{B}_{i}^{\phi}\right|\right)\right)\right\}
\end{aligned}
$$

where $F\left(\mathcal{T}^{j_{i}}\right)$ represents the current cost of the system based on the objective function in $\mathcal{P}_{T R}$ when the edge $(i, j)$ exists in the topology $\mathcal{T}$. Similarly, $F\left(\mathcal{T}^{k_{i}}\right)$ is the current cost in the system when edge $(i, k)$ exists in $\mathcal{T} . \mathcal{B}_{r}^{\phi}$ is a set containing buses having the phase $\phi$ in the subtree rooted at node $r$ and $\mathcal{B}_{r}^{\prime \phi}$ contains this information for the original subtree rooted at node $r$ prior to the switch. Eq. 20 is obtained by grouping the change in system cost into Cases 1-3 discussed in the above based on the definition of the objective function defined in $\mathcal{P}_{T R}$. 


\section{B. Decentralized Algorithm}

Now, we are equipped with the tools necessary to present the decentralized topology reconfiguration algorithm. Every bus $r$ in DN keeps track of the cardinality of its subtree $\mathcal{B}_{r}$ and the lowest bus voltage magnitude $\hat{V}_{r_{\text {min }}}$ of nodes in $\mathcal{B}_{r}$ for $\phi \in$ $\Phi_{r}$. From Eq. 20, this arrangement allows every reconfigurable bus $i \in \mathcal{R}$ to compute the change in the global cost $f_{j_{i} k_{i}}$ due to a strategy switch by accessing locally available data in $\mathcal{E}_{j_{i} A_{j_{i} k_{i}}}$ and $\mathcal{E}_{k_{i} A_{j_{i} k_{i}}}$. These buses and lines are a subset of the DN that lie well within the proximity of the switching node. This computation process is detailed in Alg. 1. If there are no constraint violations and $f_{j_{i} k_{i}}>0$, then bus $i$ will switch from node $j_{i}$ to $k_{i}$. Otherwise, the switch will not take place. These reconfiguration decisions are made at random exponentially distributed time instances defined by the parameter $\lambda$. The revision process is repeated until no further improvement to the global cost can be attained in the system.

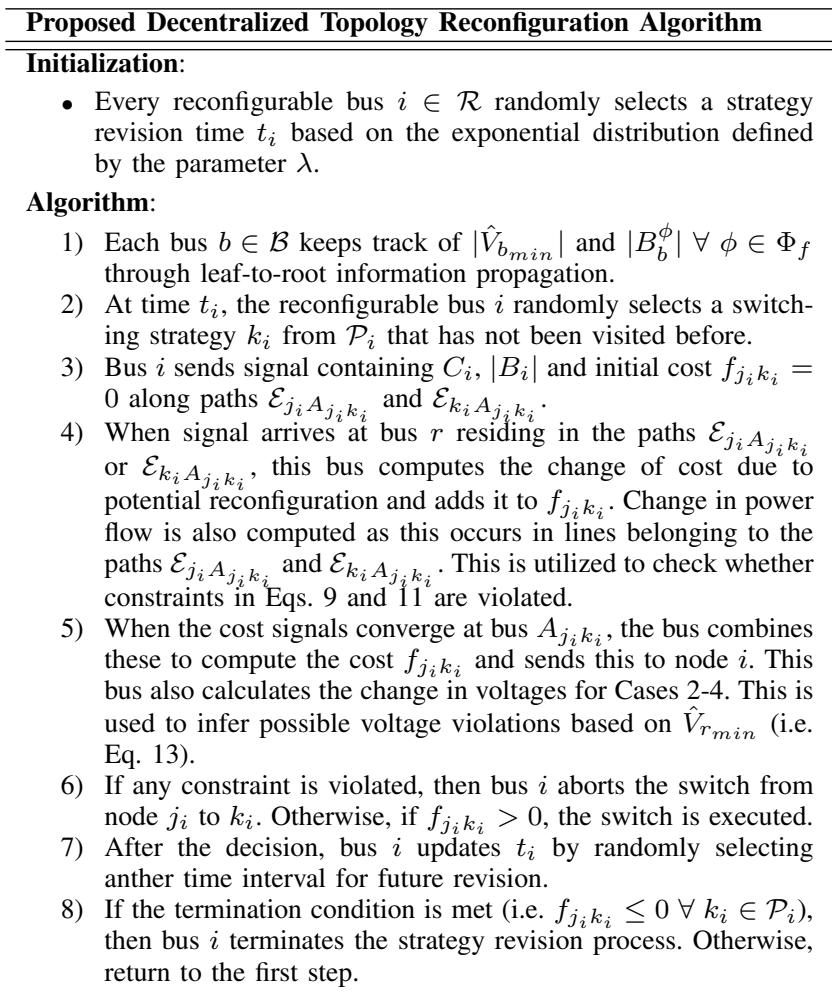

In Alg. 1, signals are exchanged amongst peers that contain information necessary to compute the global change in cost as outlined in Eq. 20 and to ensure that the system limits are heeded. These signal exchanges and iterative decision-making process outlined in the above exhibit desirable convergence properties as shown later in this section. We also derive conditions that can be checked in a decentralized manner to assess whether the resulting equilibrium is globally optimal.

\section{Switch and Branch Exchange Cases}

Prior to proceeding further, we present the two different topology reconfiguration methods considered in this paper. These are referred to as switching and branch exchange cases. In the switching case, a reconfigurable bus $i \in \mathcal{R}$ will swap its current parent with a node in $\mathcal{P}_{i}$ based on cost computations outlined earlier. In the branch exchange case considered in references such as [27], multiple switches may occur. If a reconfigurable bus $i \in \mathcal{R}$ forms an edge with a new parent in $\mathcal{P}_{i}$ without removing the old edge with the original parent, a loop is formed and this is referred to as a fundamental loop $O_{i}=\left\{\mathcal{E}_{j_{i} A_{j_{i} k_{i}}} \cup \mathcal{E}_{A_{j_{i} k_{i}} k_{i}} \cup \mathcal{E}_{k_{i} i} \cup \mathcal{E}_{i j_{i}}\right\}$. For example in Fig 3 , nodes $\left\{i, k_{i}, A_{j_{i} k_{i}}, j_{i}\right\}$ form a fundamental loop. As the DN supports only radial networks, this loop must be broken. Unlike the switching case where the edge formed with the original parent of the reconfigurable bus is removed and a new edge is formed with the incumbent parent, in the branch exchange case, any one of the edges forming the fundamental loop can be selected to be removed as long as the multiphase criteria is maintained (i.e. the number of phases supported by the potential parent nodes must be a superset of bus $r$ ). As illustrated in Fig. 3, any edge defining the fundamental loop can be removed to restore the radial structure of the DN. The edge removal that leads to the least cost is typically selected.

We transform the branch exchange case into the switching configuration by equipping all nodes with switches (not necessarily always reconfigurable) and allowing the set $\mathcal{R}$ to change over time. Specifically if $i \in \mathcal{R}$ switches to a different parent, its previous parent $j_{i}$ takes its place in $\mathcal{R}$ after the switching operation. Thus, the algorithm outlined in Sec. III-B can also be applied for the branch exchange case.

\section{Convergence and Optimality}

Repeated revisions made by individual reconfigurable buses as outlined in Sec III-B will terminate when the cost incurred by the system cannot be reduced further. Two important considerations are whether these revisions will end in finite time (i.e. convergence is guaranteed) and under what conditions will the resulting DN topology be globally optimal for $\mathcal{P}_{T R}$. To ascertain convergence and identify the optimality conditions, the decentralized topology reconfiguration problem is first formulated as a game $\mathcal{G}(\mathcal{N}, \mathcal{S}, \mathcal{V})$ where $\mathcal{N}$ is the set representing all players participating in the game, $S_{i} \in \mathcal{S}$ is the collection of strategies available to player $i \in \mathcal{N}$ (i.e. which adjacent lines to activate based on $\left.\mathcal{P}_{i}\right), V_{i} \in \mathcal{V}$ is the cost incurred by player $i \in \mathcal{N}$, and $F$ is the global cost of the game as listed in $\mathcal{P}_{T R}$. In the context of the problem considered in this paper, $\mathcal{N}$ is a finite set that represents all buses capable of reconfiguration (i.e. $\mathcal{N}=\mathcal{R}$ ), $S_{i}$ is the set of potential parents (i.e. equivalent to $\mathcal{P}_{i}$ ) which is a discrete set and $V_{i}$ is the cost change incurred by node $i$ for selecting a particular switching strategy. The global cost $F$ is then defined as the objective function of the reconfiguration problem. Furthermore, the current strategy selected by player $i$ is denoted as $s_{i} \in S_{i}$ and strategies selected by all other players (excluding player $i$ ) is $s_{-i} \in S_{-i}$. Based on our definitions of the cost function for the game and the topology reconfiguration problem, the following relation between player cost $V_{i}$ and global cost $F$ is satisfied for all $i \in \mathcal{N}$, any two strategies $x$ and $y$ available to node $i$ (i.e. $x, y \in S_{i}$ ) and any $s_{-i} \in S_{-i}$. This relation leads to Lemma 1.

$$
V_{i}\left(x, s_{-i}\right)-V_{i}\left(y, s_{-i}\right)=F\left(x, s_{-i}\right)-F\left(y, s_{-i}\right)
$$


Lemma 1. The game $\mathcal{G}(\mathcal{N}, \mathcal{S}, \mathcal{V})$ representing the topology reconfiguration problem is an exact potential game.

Proof. According to reference [34], when a game is defined so that Eq. 21 is satisfied and the set of players $\mathcal{N}$ is finite, it is classified as an exact potential game.

Next, the trajectory of the system cost (i.e. potential of the game) due to rational strategy revisions made by individual players using local costs is summarized in Lemma 2.

Lemma 2. Strategy revisions made by players one-at-a-time using local costs computed using Alg. 1 results in the monotonically decreasing potential/cost for the game $\mathcal{G}(\mathcal{N}, \mathcal{S}, \mathcal{V})$ which represents the topology reconfiguration problem.

Proof. In the topology reconfiguration problem, every agent selects a random time to revise local strategies. Since a continuous distribution is utilized to select the revision time, it is highly unlikely that these will coincide. Thus, each player will make a strategy revision one-at-a-time. Next, in the proposed algorithm, each agent $i$ will make a strategy change from $j_{i} \in \mathcal{P}_{i}$ to $k_{i} \in \mathcal{P}_{i}$ if the change in cost due to the switch is positive (i.e. $f_{j_{i} k_{i}}=F\left(\mathcal{T}^{j_{i}}\right)-F\left(\mathcal{T}^{k_{i}}\right)>0$ ) and local constraints are heeded. This implies that when a strategy change occurs, $F\left(\mathcal{T}^{j_{i}}\right)>F\left(\mathcal{T}^{k_{i}}\right)$. Let $k$ be the iteration of strategy revision, $s_{i}^{k}$ be the strategy revision made by player $i$ at iteration $k$ and $s^{k}=\left(s_{i}^{k}, s_{-i}^{k}\right)$ be the overall strategy profile in place within the system at iteration $k$. Then, it is clear that the following relation holds for the proposed topology reconfiguration game:

$$
F\left(s^{1}\right)>F\left(s^{2}\right)>F\left(s^{3}\right)>\ldots
$$

which indicates that the potential/cost of the system is decreasing for every strategy revision made by each player. This is indeed the case for the proposed topology reconfiguration algorithm as every agent considering a strategy revision will proceed with the impending revision only if the global cost in the DN decreases due to the strategy revision. Thus, at every iteration, the current cost incurred in the system will decrease until an equilibrium is reached. The sequence of strategy revisions $\zeta=\left(s^{1}, s^{2}, s^{3}, \ldots\right)$ that results in the reduction of the global cost/potential of the system is referred to as the improvement path [34].

From Lemma 2, it is clear that the strategy revisions conducted by the agents will result in the reduction of the potential of the system. The next important consideration is whether these strategy revisions will converge to an equilibrium in finite time. This is addressed by Theorem 3 presented next.

Theorem 3. The proposed algorithm will always converge to a pure-strategy Nash Equilibrium in finite time.

Proof. Since the player set $\mathcal{N}$ and discrete strategy space $\mathcal{S}$ are both finite, every improvement path $\zeta$ is a finite sequence [34]. A finite sequence will always reach an equilibrium point and this is the case with $\zeta$ as well. At all equilibrium points of $\mathcal{G}$, according to Alg. 1 no player $i$ will be able to reduce $V_{i}$ further by switching. Thus, the current state results in no regret and this is a pure-strategy Nash Equilibrium [35]. Hence, every $\zeta$ will reach a pure-strategy Nash Equilibrium in finite time.

The next important consideration is whether the Nash Equilibrium solution is also the globally optimal solution. If the strategy space is continuous, the Nash Equilibrium solution is also the optimal solution. However, for the game $\mathcal{G}$ constructed in this paper, the strategy space is discrete. Thus, to analyze the optimality of the Nash Equilibrium, we introduce the notion of independence and utilize discrete concavity to derive conditions in the DN that guarantee optimality. When fundamental loops $O_{v}$ and $O_{u}$ have no common edges, these loops are independent from one another. This notion of independence leads to Theorem 4.

Theorem 4. If all fundamental loops in the DN are independent from each other, the Nash Equilibrium resulting from the decentralized algorithm is optimal.

Proof. Consider two fundamental loops $O_{u}$ and $O_{v}$ in the DN. It can be observed from Eq. 20 that cost change from any reconfigurable bus $u$ in $O_{u}$ is a function of the following values: the difference between voltages of its potential parents $\hat{V}_{k_{u}}^{\prime}-\hat{V}_{j_{u}}^{\prime}$; its cumulative load $\mathcal{C}_{u}$; the cardinality $\left|\mathcal{B}_{r}^{\phi}\right|$ for every phase $\phi \in \Phi_{r}$ of every bus $r \in O_{u}$; and impedance $z_{r l}$ of all $(r, l) \in O_{u}$. Due to the radial structure of the DN, these values will be affected only when switching occurs in another fundamental loop $O_{v}$ in which a subset of the edges coincide with $O_{u}$. Therefore, if no edges in fundamental loops coincide with one another (i.e $O_{u} \cap O_{v}=\emptyset \forall u, v \in \mathcal{R}$ ), then altering the status of any switch within these fundamental loops will not influence the cost of one another. Thus, in this case the fundamental loops are independent from one another and the associated Nash Equilibrium will be optimal.

While the independence condition does not typically hold for all fundamental loops in existing DN networks, the condition of discrete concavity, which generalizes upon the independence condition, is applicable to a number of existing DNs and can be checked in a decentralized manner. We reformulate the discrete concavity condition presented in reference [36] for the decentralized topology reconfiguration problem. The utility function is defined as $U=-F$. The status sets for all reconfigurable buses $i \in \mathcal{R}$ is represented by $\mathcal{X} \in \mathbb{R}^{|\mathcal{R}|}$ where if bus $i$ in $|\mathcal{R}|$ is connected to its original parent, then its corresponding element $x_{i}$ in the status set $x \in \mathcal{X}$ is 1 , otherwise $x_{i}=0$. If we use $\|$.$\| to denote the first-norm, for$ any $x, y \in \mathcal{X},\|x-y\|$ is 1 if the DN configuration represented by $x$ and $y$ differs by the status of a single reconfigurable bus. Similarly, $\|x-y\|$ is 2 if the DN configuration represented by $x$ and $y$ differs by the statuses of two reconfigurable buses. The definition of the discrete concavity condition for the reconfiguration problem is listed in the following:

$$
\max _{x, y, z \in X:\|x-z\|=\|y-z\|=1} U(z)=\left\{\begin{array}{l}
>\min (U(x), U(y)), \text { if } U(x) \neq U(y) \\
\geq U(x)=U(y), \text { otherwise }
\end{array}\right.
$$

That is, the topology reconfiguration problem is discretely concave if for any two status sets $x, y \in \mathcal{X}$ such that $\|x-y\|=2$, the smaller utility value between $U(x)$ and $U(y)$ is lower than 


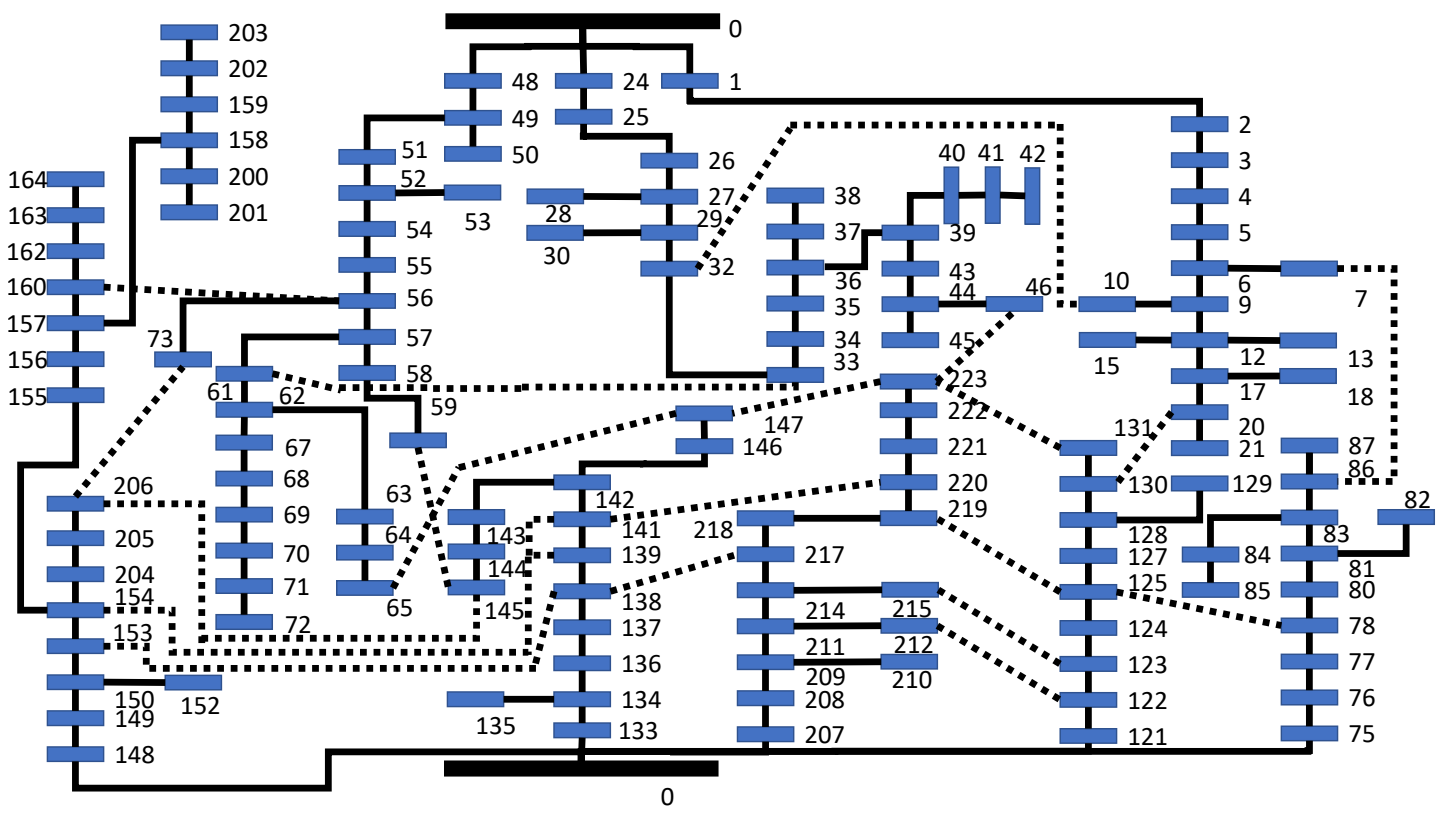

Fig. 4: Illustration of 136-21 DN feeder system.

the highest $U(z)$ that satisfies $\|z-y\|=1$ and $\|z-x\|=1$. In terms of the proposed topology reconfiguration algorithm, since strategy revisions do not occur simultaneously, when discrete concavity is satisfied, the following case cannot occur. Suppose that a strategy switch resulting in status change from $x$ to $z$ results in a lower utility (i.e. $U(z)<U(x)$ ) and then another switch resulting in status $y$ has a higher utility than that in status $x$ (i.e. $U(y)>U(x)$ ). This scenario results in the failure of the discrete concavity condition and as our algorithm considers strategy revisions that take place one at a time, it may be possible that this condition will not be explored. This leads to the following theorem.

Theorem 5. If the utility function $U=-F$ satisfies the discrete concavity condition, the equilibrium point of the topology reconfiguration algorithm will be optimal.

Proof. From Theorem 3, any equilibrium point resulting from strategy revisions conducted based on the proposed algorithm will always be locally optimal. Locally optimal solutions are proven to be globally optimal for problems that are discretely concave by reference [36].

In order to ascertain that the discrete concavity condition holds in the system, we detail how each node can perform that verification in a decentralized manner while performing local strategy revisions. If there is an improvement in cost due to a switch from $x$ to $z$, then discrete concavity is locally satisfied (i.e. $U(x)-U(z) \leq 0$ ). However, when no strategy switch will lead to an improvement in cost for node $i$, the discrete concavity condition maybe violated. In order to verify whether this is the case, it is necessary to first assess whether $O_{i}$ has overlapping edges with other fundamental loops in the system. If not, independence holds and this implies that discrete concavity is satisfied for that node. Otherwise, a reconfigurable bus located in a loop that shares an edge with the loop containing bus $i$ must be switched simultaneously with node $i$ so that $\|x-y\|=2$ where $x$ is the original state and $y$ is the state attained when two switches are made. This must be examined for all reconfigurable buses in loops that are directly adjacent to $O_{i}$. If $U(z)$ which is the utility of the switch by $i$ is lower than any $U(y)$, then the discrete concavity condition is violated. It is important to note that even if the solution is not optimal, there is no regret in the system (i.e. Nash Equilibrium is achieved).

As this check for discrete concavity is local to the bus performing the reconfiguration in both the switching and branch exchange cases, it can be readily inferred whether the DN system results in the globally optimal solution.

\section{RESUlTS}

In this section, results illustrating the performance of the proposed decentralized DN topology reconfiguration algorithm and its impact on the voltage profiles of various IEEE distribution test feeder systems for both the switching and branch exchange reconfiguration cases are presented. Comprehensive comparative results are also presented to highlight the contributions of the proposed topology reconfiguration algorithm from the existing state-of-the-art.

\section{A. Test Systems and Parameters}

To illustrate the impact of the number of buses and switches present in the DN on the performance of the proposed algorithm, simulations are conducted on various DN test feeder systems. To simplify presentation, each test system is referred to by the tuple $a-b$ where the first component, $a$, indicates the number of buses and the second component, $b$, refers to the number of tie switches (i.e. buses in the initial set $\mathcal{R}$ ) present in the DN.

For the switching case, the proposed algorithm is implemented on five radial test systems. These systems are: $33-5$, a $12.66 \mathrm{kV}$ system composed of 33 buses and 5 tie switches [27]; 
69-5, a $12.66 \mathrm{kV}$ system with 69 buses and 5 tie switches [37]; 69-7 created by randomly adding two tie switches to the 69-5 system; 69-10, created by randomly adding five tie switches to the 69-5 system; and 136-21, a $13.8 \mathrm{kV}$ Brazilian feeder system consisting of 136 buses with 21 tie switches [38]. For the branch exchange case, we consider four test systems: 33-3, created by randomly removing two tie switches from $33-5 ; 33-$ 7 , created by randomly adding two tie switches to $33-5$, and the original 33-5 and 69-5 test systems. To ensure that the presented results are unaffected by the initial topology of the test systems, 100 and 50 randomly generated initial topologies of the test systems are considered for the switching and branch exchange cases respectively. A sample of the 136-21 network is illustrated in Fig. 4 where the dotted lines represent inactive lines and the dark lines depict currently active lines.

Actual steady-state voltages and power flow resulting from topology reconfigurations are computed using the NewtonRaphson power flow algorithm in MATPOWER [39]. For analyzing the optimality of the solution computed by the algorithms under consideration, the actual optimal solution is obtained by considering all possible system topology configurations of each test system. Since these increase exponentially with the number of buses and tie switches present in the system, implementing this brute force computation on largescale test systems is not practical. This, therefore, limits the range of test systems considered for verifying optimality especially in the branch-exchange case.

\section{B. Comparison}

The performance of the proposed algorithm is first compared with the fast non-dominated sorted genetic algorithm (FNSGA) proposed recently in reference [5]. This algorithm is chosen for comparison purposes as it is associated with better convergence performance in terms of generation limits and population sizes when compared to other recently published meta-heuristic algorithms which are vastly applied for topology reconfiguration. Two main parameters associated with FNSGA are limits on the number of generations and population size of each generation. These need to be tuned for different test systems considered. Based on reference [5], generation limit and population size are set to 5 and 20 respectively for 33-5, 33-3 and 33-7 systems; 10 and 30 for 69-5, 69-7 and 69-10 systems; and 20 and 50 for the 136-21 system. The algorithm terminates when either the generation limit is met or when no changes occur in the best solution set for four consecutive generations as specified in reference [5].

\section{Performance Criteria}

In this paper, two criteria are used for measuring the performance of the proposed and FNSGA algorithms. The first criteria, the improvement factor $\mathcal{I} \in[0,1]$, is defined as the ratio of cost reduction resulting from the algorithm to the optimal cost reduction. That is, if the initial topology is defined as $(\mathcal{R}, \mathcal{P})$, solution topology from algorithm as $\left(\mathcal{R}_{\text {sol }}, \mathcal{P}_{\text {sol }}\right)$, and the optimal topology as $\left(\mathcal{R}_{\text {opt }}, \mathcal{P}_{\text {opt }}\right)$, then

$$
\mathcal{I}=\frac{\mathcal{T}(\mathcal{R}, \mathcal{P})-\mathcal{T}\left(\mathcal{R}_{\text {sol }}, \mathcal{P}_{\text {sol }}\right)}{\mathcal{T}(\mathcal{R}, \mathcal{P})-\mathcal{T}\left(\mathcal{R}_{\text {opt }}, \mathcal{P}_{\text {opt }}\right)}
$$

The second criteria, the number of iterations $I_{t}$, is defined as the number of times the state of the $\mathrm{DN}$ is evaluated before convergence. Therefore, for the proposed algorithm, $I_{t}$ is defined as the total number of revisions in the system, and for FNSGA algorithm it is defined as the number of times a Newton-Raphson power flow solution is computed.

\section{Simulations for the Switching Case}

Fig. 5a illustrates the confidence interval distribution for various test systems for both the proposed algorithm and FNSGA. Test systems 33-5, 69-5, and 69-7 are discretely concave for the switching case as per the definition presented in Eq. 23. Thus, the proposed decentralized algorithm always results in the globally optimal configuration as theoretically established in the previous section. Test systems 69-10 and 136-21 do not satisfy discrete concavity for the switching case and therefore some deviation from optimality can be expected. From Fig. 5a, it is evident that the proposed algorithm achieves an average $\mathcal{I}$ of 0.9996 with a standard deviation of 0.000465 for $69-10$, and always converges to the optimal configuration for 136-21. Thus, even in the case of non-concavity, the proposed algorithm results in a solution that is very close to optimality. However, with FNSGA, there is significant deviation from optimality as evident in the confidence intervals illustrated in Fig. 5a.

Fig. 5b illustrates the convergence rate of each algorithm. In this figure, the logarithmic scale is used to highlight the relationship between average $I_{t}$ and number of switches in the test system. While $I_{t}$ increases for both the proposed algorithm and FNSGA when the number of switches increase, this is considerably lower for the proposed algorithm in comparison to FNSGA for all test systems. For the switching case, the evolution of the improvement factor for both the proposed algorithm and FNSGA is illustrated in Fig. 5c for the 33-5 test system. The improvement ratio can be seen to progress at every iteration for the proposed algorithm with quick convergence to the optimal solution without any oscillatory behaviour and this is not the case for FNSGA.

\section{E. Simulations for the Branch Exchange Case}

The above is repeated for the branch exchange case where more nodes will have the capability to perform switching. Thus, the discrete concavity condition is less likely to hold for this particular case. As illustrated in Fig. 6a, the proposed algorithm deviates slightly from the optimal solution for all four test systems while the FNSGA exhibits significant difference. In Fig. 6b, more iterations are necessary for both algorithms as expected due to the increased number of nodes present in the system with switching capability. Nevertheless, the proposed algorithm displays faster convergence to optimality. In Fig. 6c, it is evident that the proposed algorithm once again exhibits direct convergence to the optimal solution in the 335 system while entailing more iterations than the switching case. It is clear from these results that both the proposed and FNSGA methods perform better in the switching case than the branch exchange case with the proposed algorithm resulting in solutions that are very close to optimality. As future work, we 


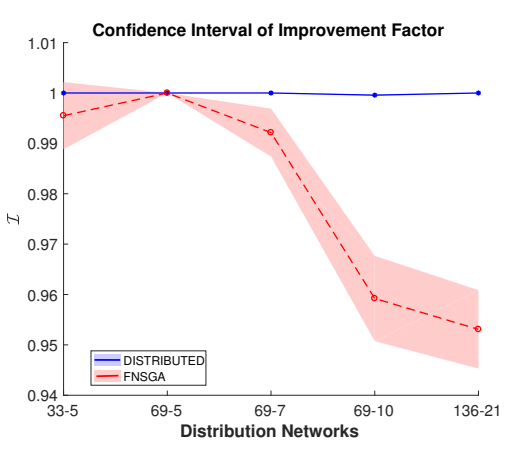

(a) Confidence interval for $\mathcal{I}$.

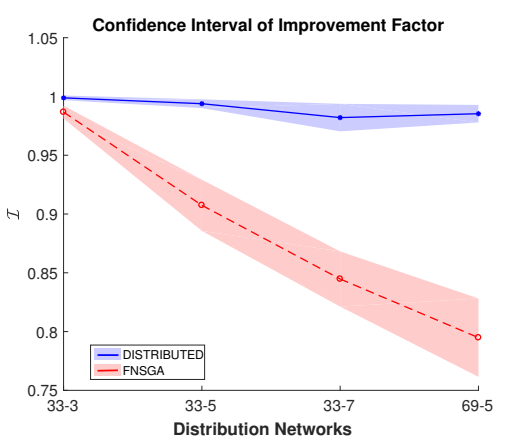

(a) Confidence interval for $\mathcal{I}$.

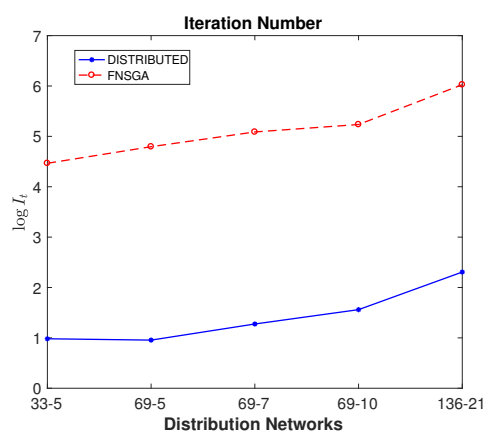

(b) Average $I_{t}$.

Fig. 5: Switching case.

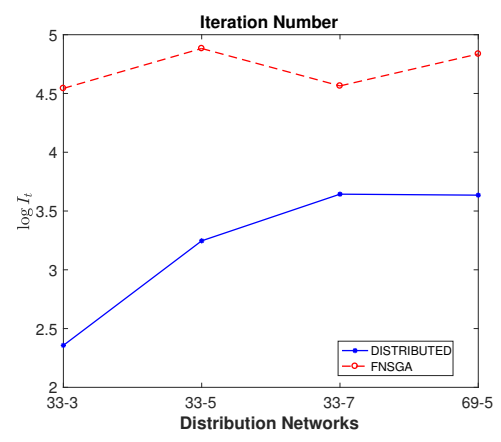

(b) Average $I_{t}$.

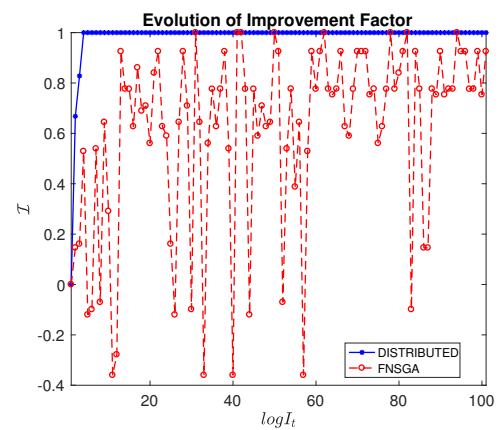

(c) Evolution of $\mathcal{I}$ for 33-5 system.

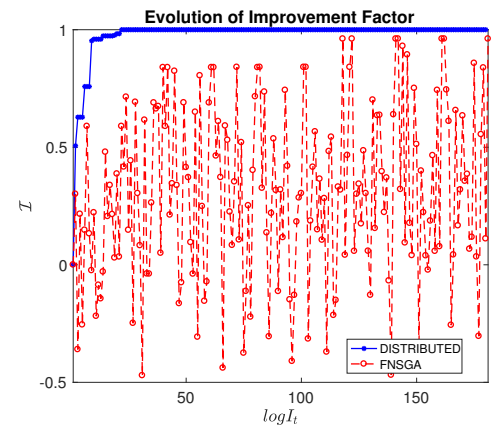

(c) Evolution of $\mathcal{I}$ for 33-5 system.

Fig. 6: Branch exchange case.

\begin{tabular}{|l|l|l|l|l|l|l|}
\hline & $\begin{array}{l}\text { Proposed } \\
\text { Algorithm }\end{array}$ & {$[\mathbf{4}],[\mathbf{5 ]},[\mathbf{7 ]},[\mathbf{1 5}]$} & {$[\mathbf{1 8}]$} & {$[\mathbf{1 2}]$} & {$[\mathbf{9 ]}$} & {$[\mathbf{1 3}]$} \\
\hline $\begin{array}{l}\text { Coordination } \\
\text { Structure }\end{array}$ & Distributed & Centralized & Distributed & Centralized & Centralized & Centralized \\
\hline $\begin{array}{l}\text { Communication } \\
\text { Extent }\end{array}$ & $\begin{array}{l}\text { Neighbouring } \\
\text { Agents }\end{array}$ & $\begin{array}{l}\text { Centralized Con- } \\
\text { troller }\end{array}$ & $\begin{array}{l}\text { All other } \\
\text { Agents }\end{array}$ & $\begin{array}{l}\text { Centralized Con- } \\
\text { troller }\end{array}$ & $\begin{array}{l}\text { Centralized } \\
\text { Controller }\end{array}$ & $\begin{array}{l}\text { Centralized } \\
\text { Controller }\end{array}$ \\
\hline $\begin{array}{l}\text { Information Re- } \\
\text { quired for Com- } \\
\text { putation }\end{array}$ & Local & Global & Global & Global & Global & Global \\
\hline $\begin{array}{l}\text { Theoretical } \\
\text { Construct }\end{array}$ & $\begin{array}{l}\text { Game } \\
\text { Theory }\end{array}$ & Meta-Heuristic & Iterative & MISOCP, MILP & QCP,SOCP & MISDP \\
\hline $\begin{array}{l}\text { Optimality Con- } \\
\text { ditions }\end{array}$ & $\begin{array}{l}\text { Independence } \\
\text { and Discrete } \\
\text { Convexity }\end{array}$ & N/A & N/A & Optimality Gap & $\begin{array}{l}\text { Optimality } \\
\text { Gap }\end{array}$ & $\begin{array}{l}\text { Optimality } \\
\text { Gap }\end{array}$ \\
\hline $\begin{array}{l}\text { Computation } \\
\text { Cost }\end{array}$ & $O(\max (|\mathcal{O}|))$ & $O\left(|\mathcal{B}|^{2}\right)$ & $O\left(|\mathcal{B}|^{2}\right)$ & $O\left(|\mathcal{B}|^{2}\right)$ & $O\left(|\mathcal{B}|^{2}\right)$ & $O\left(|\mathcal{B}|^{2}\right)$ \\
\hline $\begin{array}{l}\text { Communication } \\
\text { Cost }\end{array}$ & $O(k)$ & $O(k)$ & $O(|\mathcal{B}|)$ & $O(k)$ & $O(k)$ & $O(k)$ \\
\hline
\end{tabular}

TABLE I: Comparison table for the proposed and existing algorithms.

plan to investigate how switches can be strategically placed in the network to guarantee discrete concavity for the variability in loading expected in the system. This will allow for the application of the proposed algorithm in the switching case which will always lead to the optimal solution.

\section{F. General Comparative Analysis}

In Table I, a general comparison of proposals in existing state-of-the-art is presented. Seven features are considered in this table and these are the coordination structure (i.e. distributed/centralized), communication extent (i.e. neighbours or all agents in the system), type of information required for computations (i.e. local/global), underlying theoretical constructs applied in the proposals (e.g. game theory, heuristics, etc.), conditions for optimality (e.g. discrete concavity, duality gap), computation cost and communication cost. The first five features define general attributes of the proposals and the last two features highlight the scalability of these algorithms.

The proposed algorithm is highly scalable due to the unique approach taken to design the computation of signals and the manner in which these are exchanged amongst peers to make local actuation decisions. Since the proposed algorithm allows a switching agent to make a decision within a single traversal of a fundamental loop, its computation cost for a 
single switching decision is $O(\max (|\mathcal{O}|))$, where $\max (|\mathcal{O}|)$ is the maximum number of buses in a fundamental loop $O_{u} u \forall \mathcal{R}$ in DN. Also, since each bus in the DN only needs to communicate with its neighbours for the proposed method, its communication cost per bus for each decision is $O(k)$ where $k$ is a constant. In comparison, the centralized meta-heuristic algorithms introduced in references [4], [5], [7] require solving the power flow problem for the entire network repeatedly, with no guarantees on optimality, limited flexibility as the knowledge of the entire system is required and the possibility of single-point-of-failure issues due to the centralized construction. The computation cost of the decentralized algorithm proposed in reference [18] which uses Dijkstra algorithm to evaluate the cost of reconfiguration, is $O\left(|\mathcal{B}|^{2}\right)$ and the communication cost is $O(|\mathcal{B}|)$ as the revising agent communicates with all other agents in the system.

Another hierarchical decentralized method proposed in reference [15], which separates the DN into layers using the cut vertex method then applies the genetic algorithm to solve the problem for each layer. The computation and communication costs for this algorithm are $O\left(\max (|\mathcal{L}|)^{2}\right)$ and $O(k)$ respectively, where $\max (|\mathcal{L}|)$ is the maximum number of buses in a layer. Since a layer typically contains large number of fundamental loops, the proposed method is more scalable than this hierarchical method. The remaining three algorithms proposed in references [12], [17], [13] are centralized algorithms based on convex relaxations. Convex solvers typically entail a computational complexity of $O\left(n^{2}\right)$ where $n$ is the size of the problem (i.e. $n=\mathcal{B}$ ). The computed actuation decisions are then communicated to individual reconfigurable buses and this entails a complexity of constant time.

\section{CONCLUSIONS}

In this paper, we present a novel topology reconfiguration algorithm that allows cyber-enabled actuating nodes to adaptively react to changing conditions in the DN based on strategic signal exchanges amongst peers. The real-time and decentralized nature of the proposed algorithm increases the responsiveness of the DN to significant fluctuations which promotes resilience to congestions and cascading outages in the system. Theoretical constructs from potential games and discrete concavity along with the structural attributes of the DN have been leveraged to design local actuation and signal exchange between active nodes in the DN to exact effective coordination amongst these that guarantee convergence to Nash Equilibrium along with optimality conditions that can be checked in a decentralized manner. Moreover, the practical performance attributes of the proposed algorithm have been highlighted via simulations conducted in realistic distribution feeder networks. For future work, we plan to investigate how the switches in the DN can be placed for guaranteeing discrete concavity which will always result in the optimal solution for the proposed algorithm. We will also explore possibilities of implementing additional metrics for enhancing system performance such as considering the maximum bus voltage magnitude drop across phases instead of considering a summation of these quantities.

\section{REFERENCES}

[1] H. Farhangi, "The path of the smart grid", IEEE Power and Energy Magazine, vol. 8, no. 1, pp. 18-28, 2010.

[2] J. Glover, M. Sarma and T. Overbye, Power system design and analysis, Thomson-Engineering, 2011.

[3] P. Parikh, G. Kanabar and T. Sidhu, "Opportunities and challenges of wireless communication technologies for smart grid applications", IEEE PES General Meeting, pg 1-7, 2010.

[4] V. Roberge, M. Tarbouchi, and F. A. Okou, "Distribution system optimization on graphics processing unit", IEEE Transactions on Smart Grid, vol. 8, no. 4, pp. 1689-1699, 2017.

[5] A. M. Eldurssi, and R. M. O'Connell, "A fast nondominated sorting guided genetic algorithm for multi-objective power distribution system reconfiguration problem", IEEE Transactions on Power Systems, vol. 30 , no. 2, pp. 593-601, 2015.

[6] A. Swarnkar ,N. Gupta and K. Niazi, "Adapted ant colony optimization for efficient reconfiguration of balanced and unbalanced distribution systems for loss minimization", Swarm and Evol. Comp., vol. 1, no. 3, pp. 129-137, 2011.

[7] S. Chen, W. Hu, and Z. Chen, "Comprehensive cost minimization in distribution networks using segmented time feeder reconfiguration and reactive power control of distributed generators", IEEE Transactions on Power Systems, vol. 31, no. 2, pp. 983 - 993, 2016.

[8] C. Lee, C. Liu, S. Mehritra, and Z. Bie, "Robust distribution in network reconfiguration", IEEE Transactions on Smart Grid, vol. 6, no. 2, pp. $836-842,2015$

[9] J. A. Taylor, and F. S. Hover, "Convex models of distribution system reconfiguration", IEEE Transaction on Power Systems, vol. 27, no.3 pp. $1407-1413,2012$.

[10] L. Gan, and S. H. Low, "Convex Relaxations and Linear Approximation for Optimal Power Flow in Multiphase Radial Networks", Power Systems Computation Conference, August 18-22, 2014, Wroclaw, Poland.

[11] Q. Peng, Y. Tang, and S. H. Low, "Feeder reconfiguration in distribution networks based on convex relaxation of OPF", IEEE Transactions on Power Systems, vol. 30, no.4, 2015.

[12] R. A. Jabr, R. Singh, and B. C. Pal, "Minimum loss network reconfiguration using mixed-integer convex programming", IEEE Transactions on Power Systems, vol. 27, no. 2, 2012.

[13] Y. Liu, J. Li, L. Wu, "Coordinated Optimal Network Reconfiguration and Voltage Regulator/DER Control for Unbalanced Distribution Systems", IEEE Transactions on Smart Grid, 2018.

[14] H. Ahmadi, and J. R. Marti, "Linear Current Flow Equations With Application to Distribution Systems Reconfigurations", IEEE Transactions on Power Systems, vol. 30, no. 4, pp. 2073-2080, 2015.

[15] F. Ding, and K. A. Loparo, "Feeder reconfiguration for unbalanced distribution systems with distributed generation: A hierarchical decentralized approach", IEEE Transactions on Power Systems, vol 31, no. 2, pp. 16331642, 2016.

[16] M. Baran and F. Wu, "Network reconfiguration in distribution systems for loss reduction and load balancing", IEEE Transactions on Power Delivery, vol. 4, no. 2, pp. 1401-1407, 1989.

[17] T. Taylor and D. Lubkeman, "Implementation of heuristic search strategies for distribution feeder reconfiguration", IEEE Transactions on Power Delivery, vol. 5, no. 1, pp. 239-246, 1990.

[18] F. J. RodrÃguez, S. Fernandez, I. Sanz, M. Moranchel and E. J. Bueno, "Distributed approach for smart grids reconfiguration based on the OSPF routing protocol", IEEE Transactions on Industrial Informatics, vol. 12, no. 2, pp. 864-871, 2016.

[19] S. Sun, B. Liang, M. Dong, and J. Taylor, "Phase balancing using energy storage in power grids under uncertainty", IEEE Transactions on Power Systems, vol. 31, no. 5, pp. 3891-3903, 2016.

[20] A. Camacho, M. Castilla, J. Miret, L. G. de Vicua, and G. L. M. Andros, "Control strategy for distribution generation inverters to maximize the voltage support in the lowest phase during voltage sags", IEEE Transactions on Industrial Electronics, vol. 65, no. 3, pp. 2346-2355, 2018.

[21] V. Spitsa, X. Ran, R. Salcedo, J. F. Martinez, R. E. Uosef, F. de Lean, D. Czarkowski and Z. Zabar, "On the transient behaviour of large-scale distribution networks during automatic feeder reconfiguration", IEEE Transactions on Smart Grid, vol. 3, no. 2, pp. 887-896, 2012.

[22] Y. Tang, and S. H. Low, "Optimal placement of energy storage in distribution networks" IEEE Transactions on Smart Grid, vol. 8, No. 6, 2017.

[23] K. Orwig, et. al, "Recent Trends in Variable Generation Forecasting and Its Value to the Power System" IEEE Transactions on Sustainable Energy, vol. 6, no. 3, pp. 924-933, 2015. 
[24] N. G. Paterakis, A. Mazza, S. F. Santos, O. Erdinas, G. Chicco, A. G. Bakirtzis, and J. P. S. Catalao, "Multi-objective reconfiguration of radial distribution systems using reliability indices", IEEE Transactions on Power Systems, vol. 31, no. 2, pp. 1048 - 1062, 2016.

[25] J. Dowell and P. Pinson. "Very-short-term probabilistic wind power forecasts by sparse vector autoregression", IEEE Transactions on Smart Grid, vol. 7, no. 2, pp. 763-770, 2016.

[26] S. Low, "Convex relaxation of optimal power flow part I: Formulations and equivalence", IEEE Trans. on Cont. of Net. Sys., vol. 1, no. 1, pp.1527, 2014.

[27] M. E. Baran and F. F. Wu, "Network reconfiguration in distribution systems for loss reduction and load balancing", IEEE Transactions on Power Delivery, vol. 4, no. 1, pp. 1401-1407, 1989.

[28] L .Gan, N .Li, U .Topcu, and S .H. Low, "Optimal power flow in tree networks", 52nd IEEE Conf. on Dec. and Cont., pg. 2313-2318, 2013.

[29] R. Srinivasa Rao, K. Ravindra, K. Satish, and S. V. L. Narasimham, "Power loss minimization in distribution system using network reconfiguration in the presence of distributed generation", IEEE Transactions on Power Systems, vol. 28, no. 1, pp. 317-325, 2013

[30] H. J. Liu, W. Shi, and H. Zhu, "Decentralized dynamic optimization for power network voltage control", IEEE Transactions on Signal and Information Processing over Networks, vol. 3, No. 3, 2017.

[31] A. Abboud, F. Iutzeler, R. Couillet, M. Debbah, and H. Siguerdidjane, "Distributed production-sharing optimization and application to power grid networks" IEEE Transactions on Signal and Information Processing over Networks, vol. 2, No. 1, 2016.

[32] A. K. Sahu, S. Kar, J. M. F. Moura, and H. V. Poor, "Distributed constrained recursive nonlinear least-squares estimation: algorithms and asymptotics", IEEE Transactions on Signal and Information Processing over Networks, vol. 2, No. 4, 2016.

[33] A. Mokhtari, W. Shi, Q. Ling, and A. Ribeiro, "A decentralized second-order method with exact linear convergence rate for consensus optimization" IEEE Transactions on Signal and Information Processing over Networks, vol. 2, No. 4, 2016.

[34] D. Monderer and L. Shapley, Potential games, Games and Economic Behaviour, vol. 14, no. 1, pp. 124-143, 1996.

[35] L. Pavel, Game theory for control of optical networks, Springer Science \& Business Media, 2012.

[36] T. Ui, Discrete concavity for potential games, International Game Theory Review, vol. 10, no. 1, pp. 1-8, 2008.

[37] M. E. Baran and F. F. Wu, "Optimal capacitor placement of radial distribution systems", IEEE Trans. on Pow. Delivery, vol. 4, no. 2, pp. 725-734, 1989.

[38] J. R. S. Mantovani, F. Casari, and R. A. Romero, "Reconfiguração de sistemas de distribuição radiais utilizando o critério de queda de tensão, Revista Controle e Automação", Sociedade Brasileira de Automática,SBA, vol. 11, no. 3, pp. 150-159, 2000.

[39] R. D. Zimmerman, C. E. Murillo-SÃ $; n$ chez, and R. J. Thomas, "MATPOWER: Steady-State operations, planning and analysis tools for power systems research and education", IEEE Transactions on Power Systems, vol. 26, no. 1, pp. 12-19, 2011.

[40] P. Srikantha and D. Kundur, "Hierarchical Signal Processing for Tractable Power Flow Management in Electric Grid Networks", IEEE Transactions on Signal and Information Processing over Networks, 2018 (Early Access Article).

[41] P. Srikantha and D. Kundur, "Resilient Distributed Real-Time Demand Response via Population Games", IEEE Transactions on Smart Grid, vol. 8, no. 6, pp. 2532-2543, 2017.

[42] P. Srikantha and D. Kundur, "Real-Time Integration of Intermittent Generation With Voltage Rise Considerations", IEEE Transactions on Sustainable Energy, vol. 8, no. 3, pp. 938-952, 2017.

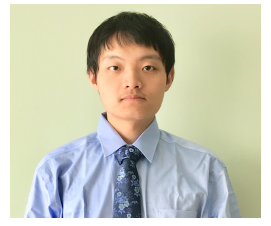

Jingyuan Liu Jingyuan Liu received his B. Sc. degree in aerospace engineering from the University of Illinois-Urbana Champaign, Champaign, IL, USA in 2015, and MSc. degree in aerospace engineering from the Purdue University, West Lafayette, Indiana, USA, in 2017. He is currently pursuing the $\mathrm{Ph} . \mathrm{D}$. degree from the Department of Electrical and Computer Engineering, Western University, London, ON, Canada. His current research interests include optimization and control of power systems and smart grid security, with a particular focus on the application of game theoretic and convex optimization techniques to distribution network reconfiguration and optimal power flow problems.

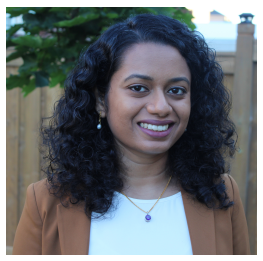

Pirathayini Srikantha is currently an Assistant Professor in the Department of Electrical and Computer Engineering at Western University. She received her B.A.Sc. degree in Systems Design Engineering from the University of Waterloo in 2009 and her M.A.Sc. degree in Electrical and Computer Engineering from the same institute in 2013. She obtained her Ph.D. degree from The Edward S. Rogers Sr. Department of Electrical and Computer Engineering at the University of Toronto in 2017. She is a certified Professional Engineer (P.Eng.) in Ontario. Her main research interests are in the areas of large-scale optimization and distributed control for enabling adaptive, sustainable and resilient power grid operations. Her work has been published in premier smart grid journal and conference venues. Her research efforts have received recognitions that include the best paper award (IEEE Smart Grid Communications) and runner-up best poster award (ACM Women in Computing). She is also actively involved in professional and social activities. She has served as the Workshop Chair, Session Chair and Technical Program Committee member in IEEE conferences. She is a reviewer in numerous IEEE transactions journals. 